\title{
Nanophotonics
}

SPIEDigitalLibrary.org/jnp

\section{Commentary: Single-molecule fluorescence spectroscopy}

Digambara Patra 


\title{
Commentary: Single-molecule fluorescence spectroscopy
}

\author{
Digambara Patra \\ American University of Beirut, Department of Chemistry, Beirut, 1107-2020, Lebanon \\ dp03@aub.edu.lb
}

Paper 11142CT received Dec. 22, 2011; revised manuscript received Jan. 2, 2012; accepted for publication Jan. 6, 2012; published online Mar. 13, 2012. [DOI: 10.1117/1.JNP.6.060301]

A focus on building sensors that can detect at the molecular level is very evident in the sensors community. Several approaches exist to detect a single atom or molecule. Fluorescence techniques have drawn enormous attention due to their selective and sensitive nature. Unlike other single-molecule detection techniques that require scanning a surface to image the morphology of an atom/molecule or atomic/molecular aggregate, in fluorescence techniques, photons emitted from a molecule are detected. Thus, fluorescence techniques are suitable for studying physical properties, interactions, and quantification rather than morphological characterization. However, morphological characterization is still possible at the single-unit level for large molecules such as proteins or aggregations when labeled with multiple fluorescent chromophores.

The focus here is on single molecule fluorescence spectroscopy (SMFS) dealing with detection and manipulation of single fluorophore level. ${ }^{1}$

In bulk spectroscopic measurements, the individual properties of molecules are hidden in ensemble averages. Detecting emission coming out from an individual molecule has many advantages over measurements done through statistical averaging. SMFS distinguishes the inhomogeneities in heterogeneous systems and investigates time-dependent processes without synchronization of molecule ensembles. It also avoids inaccuracy due to intermolecular interactions in biophysical studies. The properties of rare conformations, differences in reaction pathways, accurate distance measurement between two chromophores within the same system, emission dipole orientation in a complex system, etc., all become evident at the single-molecule level. ${ }^{2}$

A major challenge while detecting single-molecule fluorescence is background noise. A fluorescence burst, characteristic of an individual molecule, has to be spotted on top of the background associated with the solvent molecules. A simple way to reduce the background is to minimize the detection volume. A fluorescence method is very sensitive; advances in instrumentation, optics, and electronics have made it possible to record fluorescence bursts of single molecule at very diluted conditions (up to the femtoliter) by increasing the signal-to-noise ratio.

SMFS needs a laser-induced excitation source to excite a single chromophore optimally in a small volume. Generally, two different illumination set ups, as depicted in Figs. 1(a) and 1(b), are used-(a) a confocal microscope within a small detection volume or (b) a wide-field microscope with a CCD camera. Microbore capillaries and microchannels, levitated microdroplets, and hydrodynamically focused sample streams crossed with a tightly focused excitation laser beam are other possibilities.

During excitation at the electronic absorption band, a fluorescent chromophore is elevated to the excited state by absorbing a photon and then returns to the ground state by emitting a longer wavelength photon. In fluorescence, this emission is confined from the singlet excited state $\left(S_{1}\right)$. The most intriguing characteristic of an individual fluorescent molecule, shown in Fig. 1(c), is the occurrence of "off states" in its fluorescent burst. During an off state, the molecule does not emit photons on continuous irradiation with light. The occurrence of on-off states-also referred as blinking behavior-is due to the occupancy of an excited triplet state designated as $T_{1}$. Therefore, the triplet lifetime of an individual molecule can be extracted from its off-time statistics, and information can be obtained about the single molecule itself and its environment. The on-off kinetics observed in fluorescence intensity traces are assigned to molecules being either

0091-3286/2012/\$25.00 (C) 2012 SPIE 


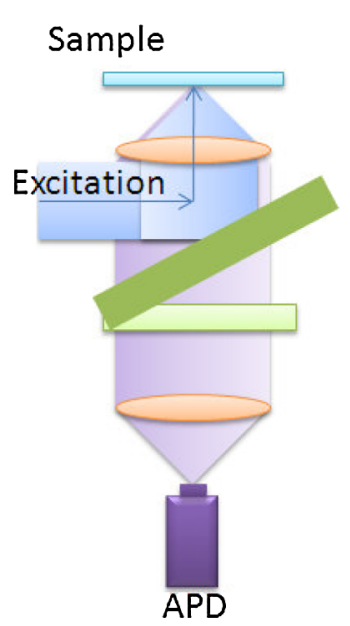

a
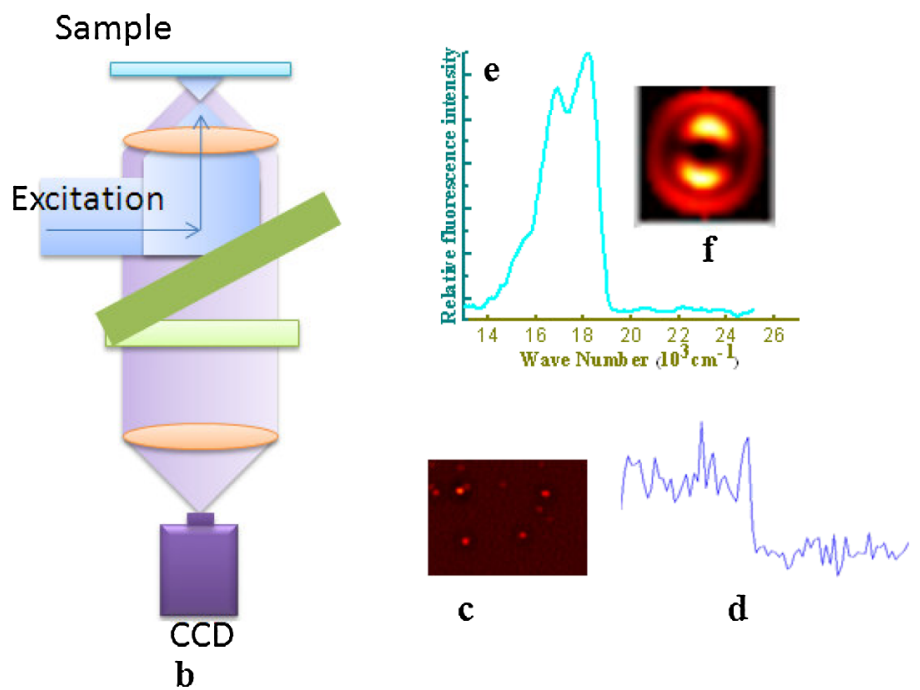

Fig. 1 (a) Confocal illumination and detection mode for SMFS, (b) wide-field illumination and detection mode for SMFS, (c) single-molecule fluorescence image of a green fluorescent protein observed by wide-field illumination and detection via CCD camera, (d) single-molecule fluorescence bleaching, (e) single-molecule spectrum of perylene imide obtained though a wavelength-calibrated CCD camera, and (f) defocused orientation image of single cy5 dye by wide-field epi-fluorescence illumination.

continuously emitting photons (on state) or occupying the triplet state (off state). This difference can help in finding out if the fluorescence is from a single molecule because in an ensemble measurement, continuous emission of photons is observed (only on state) owing to the Boltzman distribution law.

On the other hand, unlike an ensemble measurement that shows an exponential decay of fluorescence, the fluorescence from an individual molecule demonstrates single-step photobleaching as shown in Fig. 1(d)—the molecule loses its fluorescence and exhibits a permanent dark state. However, both single-molecule fluorescence photobleaching and blinking could be improved by modifying the chromophore chemically or by using external chemical scavengers. $\mathrm{N}, \mathrm{N}$-dipropyl-1,6,7,12-tetrakis(4-tert-butylphenoxy)-3,4,9,10-perylenetetra-carboxydiimide in single ultrasmall droplets of n-octane at room temperature show no distinguishable blinking in the time courses of fluorescence intensity, which is attributed to the small probabilities of the formation of the long-lived ionized state leading to the off-state of the fluorescence. ${ }^{3}$

The fluorescence spectrum of a single fluorophore can be recorded, as illustrated in Fig. 1(e), by using a wavelength-calibrated CCD camera. A change in the fluorescence emission spectrum by embedding the molecule within a tunable planar microcavity with subwavelength spacing can be studied by SMFS. ${ }^{4}$ Super-resolution fluorescence microscopy is rapidly evolving to overcome the fundamental diffraction limit of light. By relying on higher order statistical analysis of temporal fluctuations caused by fluorescence blinking or intermittency, a subdiffraction limit optical resolution can be achieved in all three dimensions-demonstrating five-fold improvement in spatial resolution. This method features significant reduction in background as demonstrated with microtubules of fibroblast cells labeled with quantum dots. ${ }^{5}$

The single-molecule trajectory analysis could give vital information about Markovian processes, such as when an enzyme molecule undergoes a turnover exhibiting no memory of its preceding turnover. Dynamical disorder, which cannot be understood by chemical kinetics, could also be explored. The spatial heterogeneity of ester cleavage rates on catalytically active crystal surfaces is revealed by SMFS. ${ }^{6}$ Association and dissociation events of a metal-complex such as the copper(II)—bipyridine chelate complex can be directly observed.

Current ensemble approaches of identifying cellular protein interactions cannot reveal physiological permutations because the same protein often participates in different complexes to exhibit diverse functionality. SMFS, when combined with the principles of a conventional pull-down assay, enables direct visualization of individual cellular protein complexes and reveals 
how many which kinds of proteins are present in the in vivo complex. ${ }^{7}$ However, observing the dynamics of single biomolecules over prolonged time periods is difficult to achieve without significantly altering the molecule through immobilization. This has been achieved by studying the photosynthetic antenna protein Allophycocyanin ${ }^{8}$ using the Anti-Brownian electrokinetic trap.

The transition moments for absorption and emission have fixed orientation within each fluorophore, and the relative angle between these moments determines the maximum measured polarization. The structural change, dynamics, conformation, colocalization, and photophysics of protein, green fluorescent proteins, and membrane fluidity are investigated by fluorescence polarization or anisotropy method. The defocused image of a fluorophore can provide information about three-dimensional (3-D) angular orientation of emission. Analysis of observed molecular emission patterns directly and noninvasively reveals true 3-D orientational dynamics of individual molecules and demonstrates that perceived molecular position is strongly dependent on orientation. Defocused emission patterns probe the 3-D orientation of the emitting chromophore in a multichromophoric system and for quantum dots, ${ }^{1}$ as shown in Fig. 1(f).

Single-pair fluorescence resonance-energy transfer (spFRET) overcomes the averaging effects of ensemble studies by the conventional FRET method, as measurements are done on single molecules freely diffusing in solution. spFRET methods are used to study intramolecular conformational changes by placing the donor and acceptor fluorescent tags on two different sites of the same macromolecule. Alternatively, intermolecular interactions are studied by attaching the donor and acceptor tags to two different macromolecules. spFRET methods measure up to three intramolecular distances and complex interaction stoichiometries of single molecules in solution and track the motion of a single molecule undergoing conformational changes.

Similarly, fluorescence imaging with 1-nm accuracy can localize the position of a single dye within a nanometer in the $x y$ plane. It is done simply by taking the point spread function of a single fluorophore excited with wide field illumination and locating the center of the fluorescent spot by a two-dimensional Gaussian fit. The step size of linear motor at nanometer accuracy could be studied using such method. ${ }^{9}$

Because of thermal motion, molecules suspended in solution undergo constant translational and rotational diffusion, and estimation of such properties can provide information about the size and shape of molecules. Fluorescence correlation spectroscopy (FCS) is done in solution by correlating the fluctuating fluorescence signal coming from a sample of fluorescent molecules at a very low concentration, which is in the concentration range for single-molecule detection. Thus, FCS is considered to be a kind of SMFS technique, in which information is extracted from molecular dynamics to understand molecular processes, such as diffusion, binding, enzymatic reactions and codiffusion, and so on. Thus, FCS has become a valuable tool for studies on living cells. Dual-focus FCS measures the velocity flow profile along the cross-section of a square-bore microfluidic channel. ${ }^{10}$

In conclusion, despite having a limited scope for a wide range of applications in comparison to conventional ensemble fluorescence measurements, SMFS has progressed as an influential tool in the following areas: chemical biology and nanosciences for understanding chemomechanical coupling in rotary and linear motor proteins; conformational change and folding of nucleic acids, proteins, polymers, and so on; assembly and association of biomolecules; translocation of DNA; gene expression; stoichiometry, diffusion, and translational motion of biomolecules; and complex emission phenomenon present in nanoclusters. In coming years, with the improvement of optics and nanoelectronics, SMFS coupled with other techniques will be essential for discovering unexplored phenomenon, particularly in complex systems and nanoscience.

\section{References}

1. D. Patra, "Single molecule studies in chemical biology and nanosciences," Curr. Chem. Biol. 2(3), 267-277 (2008), http://dx.doi.org/10.2174/187231308785739783.

2. D. Patra, "Application and developments in fluorescence spectroscopic techniques in studying individual molecules," Appl. Spectr. Rev. 43(5), 389-415 (2008), http://dx.doi.org/10 $.1080 / 05704920802108115$. 
3. M. Yasuda et al., "Fluorescence detection of single guest molecules in ultrasmall droplets of nonpolar solvent," Phys. Chem. Chem. Phys. 14(1), 345-352 (2012), http://dx.doi.org/10 $.1039 / \mathrm{c} 1 \mathrm{cp} 22207 \mathrm{~d}$.

4. A. Chizhik et al., "Tuning the fluorescence emission spectra of a single molecule with a variable optical sub-wavelength metal microcavity," Phys. Rev. Lett. 102(7), 073002 (2009), http://dx.doi.org/10.1103/PhysRevLett.102.073002.

5. T. Dertinger et al., "Fast, background-free, 3D super-resolution optical fluctuation imaging (SOFI)," Proc. Nat. Acad. Sci. USA 106(52), 22287-22292 (2009), http://dx.doi.org/10 .1073/pnas.0907866106.

6. K. Blank et al., "Watching individual enzymes at work," in Single Molecule Spectroscopy in Chemistry, Physics, and Biology, A.Gräslund, R. Rigler, and J. Widengren, eds., pp. 495-511, Springer, Berlin, Germany (2011).

7. A. Jain et al., "Probing cellular protein complexes using single-molecule pull-down," Nature 473(7348), 484-488 (2011), http://dx.doi.org/10.1038/nature10016.

8. R. H. Goldsmith and W. E. Moerner, "Watching conformational- and photo-dynamics of single fluorescent proteins in solution," Nat. Chem. 2(3), 179-186 (2010), http://dx.doi.org/ 10.1038/nchem.545.

9. R. Zhang et al., "Two-photon 3D FIONA of individual quantum dots in an aqueous environment," Nano Lett. 11(10), 4074-4078 (2011), http://dx.doi.org/10.1021/nl201225r.

10. T. J. Arbour and J. Enderlein, "Application of dual-focus fluorescence correlation spectroscopy to microfluidic flow-velocity measurement," Lab Chip 10(10), 1286-1292 (2010), http://dx.doi.org/10.1039/b924594d. 